\title{
Epithelial Cells in Patients with Fixed Orthodontic Appliances
}

\author{
Rawabi Alsalhi1, Lujain Alkhedhairi1, Sultana Alsaikhan1, Rabia Bilal2 and Shokria Ghneim³ \\ ${ }^{1}$ Dental Student, College of Dentistry, Qassim University, Buraydah, Saudi Arabia \\ ${ }^{2}$ Department of Orthodontics / Oral Pathology3, College of Dentistry, Qassim University, Buraydah, Saudi Arabia
}

\begin{abstract}
The aim of this study was to assess the morphological changes of oral mucosa epithelial cells in contact with fixed orthodontic appliances using exfoliated cytology. This study was conducted on 100 individuals that were divided into two groups (50 each). Buccal cytological smears were collected from the control group and case group at 3 to 6 months after the application of orthodontic appliances. Slides were stained with papanicolaou stain and analysed using a light microscope. In the case group, 43 out of 50 patients (86\%) exhibited morphological changes which included variable degrees of atrophy in epithelial cells; nuclear fragments and inflammatory cells infiltrate. Epithelial cells showed some morphological changes in patients with orthodontic appliances, which represent an adaptive response.
\end{abstract}

Key Words: Exfoliated cytology, Epithelial cells morphology, Fixed orthodontic therapy.

How to cite this article: Alsalhi R, Alkhedhairi L, Isaikhan S, Bilal R, Ghneim S. Epithelial cells in patients with fixed orthodontic appliances. J Coll Physicians Surg Pak 2019; 29(10):1012-4.

Orthodontic treatment is a time-consuming procedure, therefore, dental materials used in the oral cavity for both long- and short-term use should guarantee absolute safety and biocompatibility. ${ }^{1}$ Orthodontic appliances may lead to discomfort from buccal mucosa ulceration, which is a frequent complaint as a result of the friction between brackets and mucosa. Nevertheless, this discomfort diminishes after a few weeks as a result of tissue adaptation. ${ }^{2}$

Studies have described various cytoplasmic and nuclear changes after orthodontic appliance placement. These changes include variation in size and/or shape of the cell, nuclei, nuclear cytoplasmic ratio and DNA damage, which may lead to premalignant changes in particular conditions. 1-3 $^{-3}$

It was hypothesised that individuals with fixed orthodontic appliances will manifest different morphological characteristics of oral mucosa epithelial cells than normal individuals. Therefore, the aim of this study was to assess the morphological changes of oral epithelial cells in contact with fixed orthodontic appliances using exfoliated cytology.

The study was approved by the Ethical Committee of College of Dentistry, Qassim University. Written consent was taken from all the participants or their guardians for those under 18 years. The study was conducted on 100

Correspondence to: Dr. Rabia Bilal, Department of Orthodontics, College of Dentistry, Qassim University, Mullaydah,

PO Box 6700, Buraydah, Qassim 51452, Kingdom of

Saudi Arabia

E-mail: rabia.orthodontist@gmail.com

Received: November 22, 2018; Revised: March 27, 2019;

Accepted: April 08, 2019 individuals with good oral hygiene, and age range from 14-28 years, divided into two groups (50 each). The case group was composed of 50 orthodontic patients evaluated between a period of 3 and 6 months after application of orthodontic appliances. The control group included 50 individuals that were not subjected to orthodontic treatment. Exclusion criteria were individuals with systemic diseases, presence of any irritating factor such as faulty restorations or prostheses that could traumatise the mucosa, habitual biting of oral mucosa, history of previous orthodontic treatment, history of oral soft tissue lesions, and individuals having an allergy to metals.

Buccal mucosal smears were collected from the participants by stroking with a wooden tongue blade (Dukal Corporation, 9004, USA) after rinsing the mouth with water to remove excess of debris. Then, transferred to glass slides and immediately fixed with $99.9 \%$ alcohol followed by staining with papanicolaou stain. The stained smears were analysed using a light microscope (Olympus Corporation, CX21FS1, Japan).

All of the cytological stained smears were examined by one observer, who was kept blind to the case and control samples by covering the identification labels of the slides to keep the biases as minimum as possible. Five random cytological smears from each group were reexamined by the observer to check the ability of the examiner to record consistently the same changes in the same way over a period of time.

The cytosmear considered to have morphological characteristics deviated from normal, if two or more of the following features were present: variation in size and/or shape of the cells and nuclei, altered nuclear cytoplasmic ratio, and presence of inflammatory cells. 
Table I: Numbers/percentages of the cytomorphological changes in study groups.

\begin{tabular}{l|c|c|c}
\hline Variables & Case & Control & Total \\
\hline $\begin{array}{l}\text { Abnormal cells count per } 1 \times 2 \mathrm{~cm}^{2} \\
\text { (mean +SD) }\end{array}$ & $8.6 \pm 1.17$ & $4.7 \pm 2.05$ & \\
\hline Abnormality grades & & & \\
[number of subjects (\%)] & $7(14.0 \%)$ & $47(94.0 \%)$ & $54(54.0 \%)$ \\
$\quad$ None $(<5 \%)$ & $2(4.0 \%)$ & $3(6.0 \%)$ & $5(5.0 \%)$ \\
Mild $(\geq 5-<8 \%)$ & $32(64.0 \%)$ & $0(0.0 \%)$ & $32(32.0 \%)$ \\
Moderate $(\geq 8-<11 \%)$ & $9(18.0 \%)$ & $0(0.0 \%)$ & $9(9.0 \%)$ \\
Severe $(\geq 11 \%)$ & $50(100.0 \%)$ & $50(100.0 \%)$ & $100(100.0 \%)$ \\
\hline Total & \multicolumn{3}{|l}{}
\end{tabular}

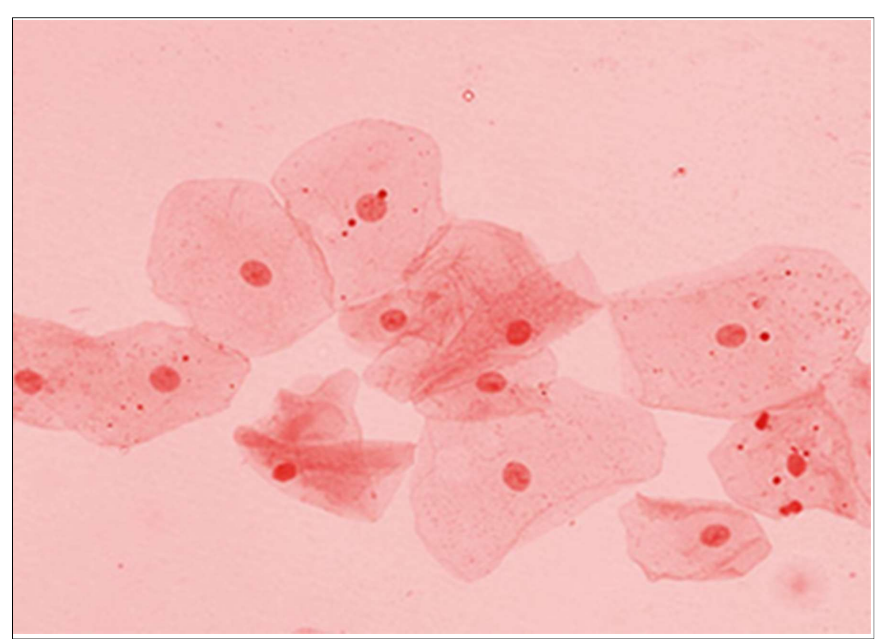

Figure 1: Epithelial cells of buccal mucosa showing mild to moderate morphological changes, including atrophy and altered nuclear cytoplasmic ratio. (PAP stain 400x).

Mean count of normal and abnormal cells were calculated in five randomly selected microscopic fields using $40 x$ objective within a square measured $1 \times 1 \mathrm{~cm}^{2}$. The two means for both types of cells were added together, to generate the total mean value of cells count for each smear. For the morphological abnormality grades of cells, the percentage of abnormal cells count in the total cells count were calculated and then classified into three grades. Any smear with $<5 \%$ abnormal cells in the examined smear was considered to be normal. But if they were between $\geq 5-<8 \%$ cells, the smear was considered to have mild abnormal cells, for $\geq 8-<11 \%$ it was moderate, and for $\geq 11 \%$ was recorded as a severe degree of morphological changes. 4

The data were analysed using SPSS version 22 (IBM SPSS Statistics) software. Independent sample t-test was used to compare means and $p \leq 0.05$ was considered significant. All data were expressed as mean \pm standard deviation (SD).

The mean age was $19.02 \pm 2.71$ in the case group, and $21.60 \pm 2.37$ in control group. Most of the participants were females, $42(84.0 \%)$ in case group and $36(72.0 \%)$ in control subjects. Numbers/percentages of the cytomorphological changes in study groups are illustrated in (Table I). There is a significant increase $(p<0.001)$ in the number of cells as well as deviation characteristics from normal morphology in orthodontic patients $(8.6 \pm 1.17)$, as compared to control subjects $(4.7 \pm 2.05)$.

Most of the orthodontic patients, i.e. 43 out of 50 , showed cytomorphological changes. Out of these 43 patients, mild to moderate morphological changes appeared in 34 patients (Figure 1), severe atrophy of epithelial cells was recorded in 9 cases, and only 2 out of these 9 cases exhibited severe acute inflammatory cells reaction in association with atrophy of epithelial cells. For the control group, only 3 cases showed a mild degree of atrophy in epithelial cells.

The oral epithelium serves as a protective barrier against the pathogens that are found in the oral cavity. ${ }^{5}$ Continuous renewal of the epithelium occurs in the deepest layers, therefore 3 to 6 months period is enough for the epithelial cells to reach the surface and manifest the adverse effects of orthodontic treatment. 5

In this study, a variation in size and/or shape of the cells, nuclei, nuclear cytoplasmic ratio and presence of inflammatory cells was observed, consistently with previous studies. ${ }^{2,3}$ There was a reduction in both, nuclear and cytoplasmic areas which are recognised as atrophic changes, and can be defined as shrinkage in the size of the cell by the loss of cell substance. ${ }^{6}$ Presence of micronuclei within the abnormal cells was also noted. Micronuclei are regarded as small additional nuclei detached from the major one, developed by the exclusion of chromosome particles or whole chromosomes lagging at mitosis, as a result of exposure of cells to cytotoxic chemical agents. ${ }^{7}$ Orthodontic patients are more vulnerable to an increased number of micronuclei due to metal ions released from the orthodontic appliances. These ions can induce genotoxic effects. ${ }^{7}$ However, Martín-Cameán et al. and de Arruda et. al. concluded that these changes were an adaptive response of the oral mucosal epithelium to mechanical friction of orthodontic appliances and were not permanent and no longer detected after removing the appliances. ${ }^{1,3}$

According to a study done by de Arruda et al., 3 there was no insignificant difference of inflammatory cells between the groups, this could be attributed to the good oral hygiene of the case group due to the monthly follow-up appointments and repeated oral hygiene reinforcement by orthodontists. In this study, no correlation between age, gender or period of treatment and cytomorphological changes was observed. Further studies with a larger sample of orthodontic patients with different types of brackets and longer follow-up are recommended to investigate the deleterious effects of orthodontic appliances and to assess the repair rate and the tendency of epithelial cells to return to normal conditions after removal of these appliances. 


\section{ETHICAL APPROVAL:}

Ethical approval from the Ethical Committee of College of Dentistry, Qassim University was obatined.

\section{PATIENTS' CONSENT:}

Consent was taken from all the participants or their guardians.

\section{CONFLICT OF INTEREST:}

Authors declared no conflict of interest.

\section{AUTHORS' CONTRIBUTION:}

RA: Design, and writting.

LA: Data collection.

SA: Data analysis.

RB: Concept, supervision, critical review, and final approval.

SG: Data analysis.

\section{REFERENCES}

1. Martín-Cameán A, Jos A, Cameán AM, Solano E, Iglesias-Linares A. Genotoxic and cytotoxic effects and gene expression changes induced by fixed orthodontic appliances in oral mucosa cells of patients: A systematic review. Toxicol Mech Methods 2015; 25:440-7.

2. Camargo E, Filho J, Filho O, Sampaio Mei R, Soares de Lima A, Tanaka $O$. A cytological analysis of the oral mucosa adjacent to orthodontic devices. European J Gen Dent 2013; 2:119-23.

3. Pinto de Arruda E, Trevilatto PC, Souza Camargo E, Ernani Cogo Woyceichoski I, Naval Machado MA, Vieira I, et al. Preclinical alterations of oral epithelial cells in contact with orthodontic appliances. Biomedical Papers 2011; 155:299-303.

4. Ahmed $\mathrm{H}, \mathrm{Al}$ Elemirri D. Assessment of oral cytological changes associated with exposure to chemotherapy and/or radio-therapy. CytoJournal 2009; 6:8.

5. Ten Cate A, Nanci A. Ten Cate's oral histology. $9^{\text {th }}$ ed. St. Louis: Elsevier; 2018. p.323-25.

6. Kumar V. Robbins basic pathology. $10^{\text {th }}$ ed. [S.I.]: ElsevierHealth Science; 2017: p.4-5.

7. Francis PG, Thomas M, Antony V, Shaloob M, Hassan KJ, Roshan G. Cytomorphometric analysis on the effects of components of orthodontic appliances on the epithelial cells of the buccal mucosa. J Int Soc Prev Community Dent 2017; 7:142-6. 